\title{
The Influences of Obesity on Central and Peripheral Gastrointestinal Responses
}

TO THE EDITOR: Recently, I read a treatise conducted by Zhang et $\mathrm{al}^{1}$ with great interest. In this issue, the authors explored if there are alterations in gastric motor function by checking myoelectrical activity, gastric emptying, autonomic functions and central neuronal responses to gastric stimulations, in diet-induced obese (DIO) rat model which especially showed significant similarity of affected biological pathways with human obesity.

First, they showed that gastric emptying was accelerated in DIO rats compared to the control ones. With this result, the authors concluded that accelerated gastric emptying increased food intake due to rapid loss of satiety. However, there have been several reports which showed opposite results from human data. ${ }^{2,3}$ Therefore, direct application of this hypothesis to human being should be considered carefully. The result of this study which showed discrepancy between myoelectrical activity and gastric emptying suggests that further studies for elucidating the mechanism of accelerated gastric transit in DIO rats are mandatory.

Second, autonomic function tests using heart rate variability showed impaired postprandial modulation of the autonomic functions in DIO group. Significant variability in postprandial heart rate, observed in control rat was not found in DIO rat. With this result, the authors suggested that impaired postprandial autonomic response is very important for the development of obesity. However, it is uncertain whether the impaired postprandial autonomic dysfunction is a cause or an effect of obesity and further studies are necessary.

Finally, the authors found that the ventromedial hypothalamus neurons in the DIO rats were more resistant to both gastric distension and gastric electrical stimulation compared to control rats. The author's group has studied about the effects of gut stimulation on neuronal activity changes in the central nervous system and reported that gastric electrical stimulation activated gastric distention-responsive neurons in the ventromedial hypothalamus, and that the effect was associated with the strength of stimulation in normal weight rats. ${ }^{4-6}$ In this issue, the authors applied this experiment on DIO rats. Although there are several hypotheses to explain the neurobiological mechanisms such as leptin resistance or dysfunction of ghrelin signaling system, ${ }^{7,8}$ there has been no report which showed decreased hypothalamic response to gastric stimulation in obese animal model, and these findings may be novel if the communication mechanism between peripheral and central pathway can be elucidated.

In spite of several limitations commented above, this study seems to be quite helpful in understanding the alterations in peripheral, autonomic and brain-gut interactions which are important in the pathogenesis of obesity.

Kyung Sik Park

Department of Internal Medicine Keimyung University School of Medicine, Daegu, Korea

1. Zhang J, Sha W, Zhu H, Chen JD. Blunted peripheral and central responses to gastric mechanical and electrical stimulations in diet- induced obese rats. J Neurogastroenterol Motil 2013;19:454-466.

2. Park MI, Camilleri M, O'Connor H, et al. Effect of different macronutrients in excess on gastric sensory and motor functions and appetite in normal-weight, overweight, and obese humans. Am J Clin Nutr 2007;85:411-418.

3. Horowitz M, Collins PJ, Cook DJ, Harding PE, Shearman DJ. Abnormalities of gastric emptying in obese patients. Int J Obes 1983; 7:415-421.

4. Qin C, Chen JD, Zhang J, Foreman RD. Characterization of T9-T10 spinal neurons with duodenal input and modulation by gastric electrical stimulation in rats. Brain Res 2007;1152:75-86.

5. Tang M, Zhang J, Chen JD. Central mechanisms of gastric electrical stimulation involving neurons in the paraventricular nucleus of the hypothalamus in rats. Obes Surg 2006;16:344-352.

6. Sun X, Tang M, Zhang J, Chen JD. Excitatory effects of gastric electrical stimulation on gastric distension responsive neurons in ventromedial hypothalamus (VMH) in rats. Neurosci Res 2006;55:451-457.

7. Bouret SG, Gorski JN, Patterson CM, Chen S, Levin BE, Simerly RB. Hypothalamic neural projections are permanently disrupted in diet-induced obese rats. Cell Metab 2008;7:179-185.

8. Gorski JN, Dunn-Meynell AA, Levin BE. Maternal obesity increases hypothalamic leptin receptor expression and sensitivity in juvenile obesity-prone rats. Am J Physiol Regul Integr Comp Physiol 2007;292:R1782-R1791.

\section{Conflicts of interest: None.}

